\title{
Eficacia del timol sobre Varroa sp y Nosema sp en colmenas utilizadas para fecundación en México
}

\author{
Loeza, C.H. ${ }^{1}$; Salgado, M.S. ${ }^{2}$; Avila, R.F. ${ }^{3}$; Escalera, V.F.; Carmona, C.A. ${ }^{2}$ \\ ${ }^{1}$ Cs. Biolog. Agropec. Univ. Autón. Nayarit, Xalisco, México. \\ ${ }^{2}$ Unid. Med. Vet. Zoot. Nayarit, ${ }^{3}$ Univ. Guanajuato, México. \\ E-mail: henryloeza_21@yahoo.com
}

\begin{abstract}
Resumen
Loeza, C.H.; Salgado, M.S.; Avila, R.F.; Escalera, V.F.; Carmona, C.A.: Eficacia del timol sobre Varroa sp y Nosema sp en colmenas utilizadas para fecundación en México. Rev. Vet. 31: 2, 202-205, 2020. Actualmente existe la necesidad de utilizar productos alternativos de origen natural que no afecten negativamente la productividad de las colmenas y que reduzcan la presencia de diversos patógenos. El timol es considerado como el producto natural más utilizado para el tratamiento de la varroasis en todo el mundo y potencialmente eficaz como nosemicida. Por lo tanto, el objetivo de esta investigación fue estudiar el impacto del timol sobre el control de la varroasis y nosemosis en colmenas para la formación de núcleos de fecundación de abejas reina (Apis mellifera). Se utilizaron 65 colmenas en las cuales se cuantificó el nivel de infestación para Varroa, nivel de infección para Nosema y las prevalencias de ambas enfermedades en la población antes y después del tratamiento, basado en 4 aplicaciones de timol al $20 \%$ en azúcar glass con intervalo de 7 días. La prevalencia de la varroasis disminuyó al igual que el nivel de infestación post tratamiento $(p<0,05)$. Por el contrario, la prevalencia de la nosemosis $(p>0,05)$ y el nivel de infección de Nosema fueron mayores después del tratamiento $(\mathbf{p}<0,05)$. Se concluye que en las colmenas para la formación de núcleos de fecundación de abejas reina, el timol es eficaz contra varroasis pero no contra nosemosis.
\end{abstract}

Palabras clave: abejas, patógenos, timol, varricida, nosemicida, prevalencia.

\begin{abstract}
Loeza, C.H.; Salgado, M.S.; Avila, R.F.; Escalera, V.F.; Carmona, C.A.: Efficacy of timol on the Varroa sp and Nosema sp in beehives used for fecundation in Mexico. Rev. Vet. 31: 2, 202-205, 2020. Nowadays, there is a need to identify alternative natural products that do not negatively affect the hive's productivity and that reduce the presence of various pathogens. Thymol is considered the most natural acaricide against varroasis worldwide, with potential nocemicide effect. Therefore, the objective of this research was to study the impact of thy mol on the presence of varroasis and nosemosis on queen mating nucleus hives (Apis mellifera). Sixty five hives were used, in which the level of infestation for Varroa $s p$, the level of infection for Nosema $s p$, and the prevalence of both diseases in the population before and after the treatment were determined, based on 4 applications of $20 \%$ thy mol in icing sugar with a 7-day interval. The prevalence of varroasis and level of Varroa $s p$ infestation decreased after treatment $(p<0.05)$. In contrast, the prevalence of nosemosis $(p>0.05)$ and level of Nosema sp infection were higher after treatment $(\mathrm{p}<0.05)$. It can be concluded that, in hives destined for the production of queen mating nucleus, thy mol is effective against varroasis but not against nosemosis.
\end{abstract}

Key words: bees, pathogens, thymol, varricide, nocemicide, prevalence.

\section{INTRODUCCIÓN}

La varroasis es considerada como la enfermedad de mayor amenaza para la apicultura ya que provoca la muerte de las colmenas infestadas dentro de los 2 a

Recibido: marzo 2020 / Aceptado: junio 2020 Proyecto SIP 17-228, Univ. Autón. Nayarit, México, financiado por PRODEP, PTC 2017
4 años de iniciada la infestación. En este contexto ningún otro patógeno ha tenido un impacto durante tanto tiempo en la apicultura ${ }^{6}$.

La nosemosis es causada por los hongos Nosema apis o Nosema ceranae. Estos microsporidios se reproducen rápidamente dentro de las células intestinales, ejerciendo un estrés energético severo en las abejas ${ }^{5,17}$. Ello afecta negativamente la productividad, supervivencia y longevidad, suprimiendo el sistema inmunita- 
rio y aumentando la vulnerabilidad de las abejas para padecer otras enfermedades ${ }^{10,20}$.

Varroasis y nosemosis son consideradas las enfermedades con mayor repercusión en las colmenas productoras de miel. Pese a ello no se ha descrito información de estas dos enfermedades en otros asentamientos productivos, ni el efecto de tratamientos.

El timol es un acaricida derivado del tomillo. Es una sustancia natural de bajo impacto ambiental, baja toxicidad y no produce resistencia en Varroa ${ }^{1,27}$, permitiendo su uso para el control de Varroa en la apicultura orgánica, según el reglamento de la Unión Europea $\mathrm{N}^{\circ} 834 / 2007^{19}$. Por estas razones, el timol se utiliza en concentraciones que van desde $9 \mathrm{~g}$ hasta $32 \mathrm{~g}$ por colmena ${ }^{9,11}$.

Siendo el varricida natural más utilizado en la apicultura, los apicultores de la región emplean el timol en forma empírica al $20 \%$ para el tratamiento contra varroasis ${ }^{25}$. El timol suprime el desarrollo de la vesícula de Nosema en las orugas de Helicoverpa armigera ${ }^{4}$ e inhibe el crecimiento de bacterias y hongos patógenos ${ }^{12,22}$. En este sentido el timol ha sido utilizado en laboratorio como tratamiento exitoso contra Nosema ${ }^{8,28}$, por lo cual -en vida libre- puede constituir una alternativa en el control de ambas enfermedades (varroasis y nosemosis).

Los núcleos de fecundación tienen la función primordial de albergar a las reinas vírgenes durante el periodo de apareamiento, entonces las colmenas formadoras de núcleos de fecundación influyen en la salud de las colmenas donde las reinas serán liberadas.

El diagnóstico y el control de Varroa y Nosema son prácticas comunes en la apicultura pues influyen en la rentabilidad de las colmenas ${ }^{15,16}$, pero hasta donde sabemos no existen reportes que cuantifiquen y traten las dos enfermedades en las colmenas que son utilizadas para la formación de núcleos de fecundación (CFNF). Por ello surgió nuestra intención de evaluar el tratamiento convencional 1:5 (timol y azúcar glass) sobre la presencia de Varroa y Nosema en las CFNF.

\section{MATERIAL Y MÉTODOS}

Ubicación del área experimental. La investigación se realizó en el Municipio de Tepic, Nayarit (México), ubicado a $21^{\circ} 51^{\prime}$ y $21^{\circ} 24^{\prime}$ latitud norte, $104^{\circ} 34^{\prime}$ y $105^{\circ} 05^{\prime}$ longitud oeste, a $915 \mathrm{~m}$ sobre el nivel del mar. En la zona predomina el clima cálido sub-húmedo con lluvias en verano y el semicálido subhúmedo con lluvias en invierno. La precipitación media anual es de $1.121 \mathrm{~mm}$ y la temperatura promedio es de $21^{\circ} \mathrm{C}^{26}$.

Unidades experimentales. Se muestrearon 5 apiarios con un total de $65 \mathrm{CFNF}$, o sea colmenas cuya principal función es donar bastidores con cría y abejas jóvenes, con la finalidad de poblar colmenas para la fecundación de abejas reinas vírgenes. Las CFNF están sometidas a la constante pérdida de población, por lo cual no se utilizan para la producción de miel, polen, propóleos ni jalea real. Ellas operan para mantener la salud de las colmenas y para garantizar el buen desarrollo de las reinas, que son criadas en los núcleos de fecundación.

Preparación y aplicación del timol. Se utilizó timol cristal previamente pulverizado, con una pureza del $99 \%$. El tratamiento consistió en la mezcla de timol y azúcar glass 1:5. Para la aplicación se prepararon paquetes con $40 \mathrm{~g}$ de la mezcla, la cual fue esparcida en cuadros de papel de $20 \times 20 \mathrm{~cm}$ sobre la cámara de cría. El tratamiento se aplicó en 4 ocasiones con intervalos de 7 días.

Obtención de muestras y procesamiento en laboratorio. Se recolectaron aproximadamente 300 abejas por colmena, tomadas entre el tercer y cuarto bastidor de la cámara de cría., colocándose recipientes con alcohol al 70\%. Las muestras se colectaron en el día 0 al principio del tratamiento y en el día 28 , siete días después del último tratamiento. La cuantificación de varroas se realizó por la técnica de Dej Jong ${ }^{7}$ y el nivel de infestación de Varroa se determinó dividiendo el número de ácaros encontrados entre el número de abejas observadas y el resultado multiplicado por 100 . La visualización de nosemas fue realizada por la técnica de Cantwell y el nivel de infección de Nosema se determinó dividiendo la cantidad de esporas observadas entre 80 y se multiplicó por 4 millones ${ }^{21}$.

Prevalencia y eficacia del tratamiento. La prevalencia de ambas enfermedades se determinó multiplicando el número de colmenas con presencia del patógeno (Varroa o Nosema) por 100 y dividido entre el total de colmenas evaluadas ${ }^{3}$. El porcentaje de eficacia se determinó mediante la resta del total de patógenos encontrados inicialmente menos el total de patógenos encontrados después del tratamiento, dividido entre el total de patógenos encontrados inicialmente y multiplicado por $100^{13,23}$. El índice de aumento se determinó mediante la resta del índice final menos el índice inicial dividido entre índice final y multiplicado por 100.

Análisis estadístico. Las diferencias de las prevalencias y los niveles de infestación de Varroa y Nosema antes y después de la aplicación de timol se realizó mediante una comparación de medias con el test t-Student, utilizando el Software Statistical Package for the Social Sciences (SPSS) versión $20.00^{24}$.

\section{RESULTADOS Y DISCUSIÓN}

La efectividad del timol contra Varroa ha sido demostrada por autores que utilizando $10 \mathrm{~g}$ de timol por colmena obtuvieron el $92,2 \%$ de eficacia ${ }^{23}$. Otros emplearon $12,5 \mathrm{~g}$ obteniendo $92,1 \%$ de eficacia ${ }^{27}$. Finalmente, algunos obtuvieron un $82 \%$ de eficacia con $32,12 \mathrm{~g}$ por colmena ${ }^{18}$. 
Tabla 1. Efecto del tratamiento con timol sobre la cuantificación de Varroa y Nosema en CFNF.

\begin{tabular}{lccccccc}
\hline periodo & \multicolumn{9}{c}{ Varroa } & \multicolumn{3}{c}{ Nosema } \\
\cline { 2 - 8 } & TC & $\mathrm{C}+$ & PV $(\%)$ & NIVC & $\mathrm{C}+$ & PN(\%) & NINC \\
\hline pre-trat. & 65 & 63 & $96,92 \mathrm{a}$ & $6,06 \pm 0,68 \mathrm{a}$ & 50 & $76,92 \mathrm{a}$ & $23,5 \pm 38,0 \times 10^{3} \mathrm{a}$ \\
\hline pos-trat. & 65 & 34 & $56,92 \mathrm{~b}$ & $0,84 \pm 0,14 \mathrm{~b}$ & 54 & $83,08 \mathrm{a}$ & $73,4 \pm 11,0 \times 10^{4} \mathrm{~b}$ \\
\hline
\end{tabular}

CFNF: colmenas que son utilizadas para la formación de núcleos de fecundación; TC: total de colmenas; $\mathrm{C}+$ : colmenas positivas; $\mathrm{PV}$ : prevalencia de Varroa; $\mathrm{PN}$ : prevalencia de Nosema; NIVC: nivel de infestación de Varroa por colmena; NINC: nivel de infección de Nosema por colmena; ( $a, b)$ letras diferentes por columna indican diferencias significativas $(p<0,05)$. Trat.: tratamiento.

En el mismo sentido, de acuerdo a un test t-Student, los resultados del presente experimento muestran que utilizando $8 \mathrm{~g}$ de timol por colmena, la eficacia contra Varroa post tratamiento fue de $85,07 \%$, de 809 a 120 ácaros de Varroa totales $(\mathrm{p}<0,05)$ y el nivel de infestación de Varroa presentó una disminución post aplicación de timol de $6,06 \pm 0,68$ a $0,84 \pm 0,14$ ácaros $/ 100$ abejas $(p<0,05)$.

Del mismo modo, el test t-Student indicó una disminución significativa de la prevalencia de la varroasis en las colmenas evaluadas, del $96,92 \%$ pretratamiento al $56,92 \%$ post-tratamiento de timol, reduciendo la prevalencia en un $41,27 \%(\mathbf{p}<0,05)$ (Tabla 1). Consideramos que la aplicación de $8 \mathrm{~g}$ de timol en base sólida, es tan eficiente para el control de la varroasis como las preparaciones en gel y jarabe utilizadas en otros trabajos $18,23,27$.

Contrariamente a la efectividad del timol contra la varroasis, la prevalencia de la nosemosis fue mayor en $7,4 \%$, de un $76,92 \%$ pre-tratamiento a $83,08 \%$ posttratamiento $(\mathrm{p}>0,05)$. Asimismo, el nivel de infección de Nosema aumentó de 23,5 $\pm 38,0 \times 10^{3}$ a 73,4 $\pm 11,0 \times$ $10^{4}$ esporas/abeja, por lo cual se observó un incremento de $67,92 \%(\mathrm{p}<0,05)$ (Tabla 1$)$.

En este sentido el timol no demostró ser efectivo en el control de la nosemosis, pero el aumento del nivel de infección no fue numéricamente suficiente para detectar la enfermedad clínica en las colmenas. De acuerdo a las referencias consultadas, no existe evidencia en la literatura que sustente la eficacia del timol como tratamiento contra Nosema en vida libre.

En condiciones controladas de laboratorio, donde las abejas inoculadas fueron mantenidas bajo la periódica ingesta de timol, los resultados indicaron que el timol redujo en un $40 \%$ la cantidad de Nosema con concentraciones de 4, 40 y $400 \mu \mathrm{M}^{8}$. Asimismo, se han observado efectos similares con concentraciones de timol de $0,12 \mathrm{mg} / \mathrm{g}$, encontrándose $8,8 \%$ después de 25 días post-tratamiento ${ }^{14}$. Del mismo modo, otros investigadores ${ }^{28}$ descubrieron que cuando se alimenta a las abejas con Nosema y con timol a una concentración de $0,1 \mathrm{mg} / \mathrm{g}$ ya sea en jarabe o caramelo, la infección se reduce un $50 \%$.

Los resultados obtenidos fueron contradictorios, pues en el presente estudio se utilizaron cantidades superiores $(1,331 \mathrm{mM})$ de timol a lo reportado ${ }^{8,28}$, en- contrando un incremento de la enfermedad en las colmenas, posiblemente por las condiciones en que varios investigadores realizaron sus indagaciones ${ }^{14,19,28}$, mientras que esta investigación se realizó a nivel de campo, en condiciones naturales, permitiendo la entrada y salida de las abejas, la obtención de alimentos ad limitum y el intercambio de secreciones entre las abejas obreras, razones por las cuales los resultados en vida libre difieren, pues existen factores que permitieron el mantenimiento y desarrollo de Nosema.

Creemos que después del tratamiento de timol contra Varroa mejoraron las condiciones de las abejas, aumentando la cantidad de hemocitos y mejorando el metabolismo de las proteínas de la hemolinfa ${ }^{6}$. Dado que -como consecuencia- se favoreció el desarrollo de Nosema, es posible que en experimentos controlados no se pueda observar este fenómeno, debido a la limitación por una dieta específica y controlada, lo cual posiblemente limita el crecimiento del hongo dentro de los hospedadores.

$\mathrm{Si}$ se tratan las abejas con timol, la cantidad de $\mathrm{Va}$ rroa disminuye y no hay competencia entre patógenos por los nutrientes, aumenta la condición corporal de las abejas y entonces Nosema prolifera ${ }^{2}$. De lo anterior se puede hipotetizar que el incremento de la prevalencia de nosemosis y el nivel de infección de Nosema después del uso del timol fueron efectos secundarios debidos a la disminución de la presencia de varroasis.

La ineficacia del timol contra la nosemosis también se constató en trabajos previos donde se concluyó que el timol no es limitante para el desarrollo de Nosema al incorporarse $15 \mathrm{mM}$ y $30 \mathrm{mM}$ de timol en la dieta de orugas, obteniendo un decremento del $53,8 \%$ y $59.2 \%$ de Nosema vespula respectivamente ${ }^{4}$.

Agradecimientos. A los apicultores del Sistema Producto Apícola de Nayarit, por permitir esta investigación y dedicar tiempo y esfuerzo para realizar la obra. Al Consejo Nacional de Ciencia y Tecnología por otorgar el financiamiento y una beca.

\section{REFERENCIAS}

1. Adamczyk $\mathbf{S}$ et al. 2005. Evaluation of residues of essential oil components in honey after different anti-Varroa treatments. J Agric Food Chem 53: 10085-10090.

2. Alaux C et al. 2010. Interactions between Nosema microspores and a neonicotinoid weaken honey bees (Apis mellifera). Environ Microbiol 12: 774-782.

3. Amiri E, Strand MK, Rueppell O, Tarpy DR. 2017. Queen quality and the impact of honey bee diseases on queen health: potential for interactions between two major threats to colony health. Insects $8: 48$. 
4. Bulacio CN, Basualdo M, Eguaras M. 2010. Actividad varroacida del timol en colonias de Apis mellifera de la Provincia de Santa Fe. In Vet 12: 85-90.

5. Cantwell GE. 1970. Standard methods for counting $\mathrm{No}$ sema spores. Amer Bee J 110: 222-223.

6. Conte Y, Ellis M, Ritter W. 2010. Varroa mites and honey bee health: can Varroa explain part of the colony losses? Apidologie 41: 353-363.

7. Costa C, Lodesani M, Maistrello L. 2010. Effect of thymol and resveratrol administered with candy or syrup on the development of Nosema ceranae and on the longevity of honeybees in laboratory conditions. Apidologie 41: 141150 .

8. Dejong D, Dejong P, Goncalves L. 1982. Weight loss and other damage to developing worker honeybees from infestation with Varroa jacobsoni. J Apicult Res 21: 165-167.

9. Espinosa LG, Guzmán E. 2007. Effectiveness of two natural miticides, formic acid and thymol, for control of the mite Varroa destructor in honey bees (Apis mellifera) in Villa Guerrero, Mexico. Vet Mexico 38: 9-19.

10. Fernández EA, Zavala HJ, Romero CR. 2010. Atlas climático digital de México. Centro de Ciencias de la Atmósfera, UNAM. Available online: http://atlasclimatico. unam. $\mathrm{mx} / \mathrm{atlas} / \mathrm{kml}$.

11. Floris I, Satta A, Cabras P, Garau VL, Angioni A. 2004. Comparison between two thymol formulations in the control of Varroa destructor: effectiveness, persistence, and residues. J Econom Entomol 97: 187-191.

12. Heeve JP et al. 2016. Evaluation of Fumagilin-B and other potential alternative chemotherapies against Nosema ceranae-infected honeybees (Apis mellifera) in cage trial assays. Apidologie 47: 617-630

13. Higes M et al. 2008. Detection of infective Nosema ceranae (Microsporidia) spores in corbicular pollen of forager honeybees. J Invertebr Pathol 97: 76-78.

14. Imdorf A, Bogdanov S, Kilchenmann V. Maquelin C. 1995. Apilife var: a new varroacide with thymol as the main ingredient. Bee World 76: 77-83

15. Juven B, Kanner J, Schved F, Weisslowicz H. 1994. Factors that interact with the antibacterial action of thyme essential oil and its active constituents. J Appl Microbiol 76: 626-631
16. Llorente J, Higes M, Suárez M. 1996. Actas del II Congreso de la Sociedad Española de Agricultura Ecológica, Pamplona: orgprints.org/29757/1/actas-pamplona-vd.pdf.

17. Mahmoud AL. 1999. In hibition of growth and aflatoxin biosynthesis of Aspergillus flavus by extracts of some egyptian plants. Letters Appl Microbiol 29: 334-336.

18. Maistrello L et al. 2008. Screening of natural compounds for the control of Nosema disease in honeybees (Apis mellifera). Apidologie 39: 436-445.

19. Montano L, Guzman NE. 2007. Effectiveness of two natural miticides, formia acid and thymol, for control of the mite Varroa destructor in honey bees (Apis mellifera) in Villa Guerrero, Mexico. Vet Mexico 38: 9.

20. Moreno AA, López MS, Corcho BA. 2000. Principales medidas en epidemiología. Salud Públ Méx 42: 337-348.

21. Ravoet J et al. 2013. Comprehen sive bee pathogen screening in Belgium reveals Crithidia mellificae as a new contributory factor to winter mortality. PLoS One 8: e72443.

22. Reyes FR. 2016. Efectividad de cuatro acaricidas en el control del ácaro (Varroa destructor) en abejas. http://repositorio.lamolina.edu.pe/handle/UNALM/2755.

23. Rice RN. 2001. Nosema disease in honeybees. Genetic variation and control (Australian Report). Res \& Develop Corp 1: 46

24. SPSS Inc. 1999. Statistical package for the social sciences. Chicago, Illinois, USA. https://www.academia.edu.

25. Unión Europea Diario Oficial. 2007. Regl. Unión Europea $\mathrm{N}^{\circ} 834 / 2007$. https://eur-lex.europa.eu/legal-content/ es/TXT/? uri=CELEX:32007R0834.

26. Viollon C, Chaumont JP. 1994. Antifungal properties of essential oils and their main components upon Cryptococcus neoformans. Mycopathologia 128: 151-153.

27. Williams BA. 2009. Unique physiology of host-parasite interactions in micros- poridia infections. Cellular Microbiol 11: 1551-1560.

28. Williams GR., Shutler D, Burgher KL, Rogers RE. 2014. Infra-population and community dynamics of the parasites Nosema apis and Nosema ceranae, and consequences for honey bee (Apis mellifera) hosts. PLoS One 9: e99465. 